\title{
The Use of Image Smoothness Estimates in Speeding Up Fractal Image Compression
}

\author{
Tomas Žumbakis ${ }^{1}$ and Jonas Valantinas ${ }^{2}$ \\ ${ }^{1}$ Kaunas University of Technology, LT-51368 Kaunas, Lithuania \\ t.zumbakis@vb.1t \\ ${ }^{2}$ Kaunas University of Technology, LT-51368 Kaunas, Lithuania \\ jonas.valantinas@ktu. It
}

\begin{abstract}
The paper presents a new attempt to speed up fractal image encoding. The range blocks and corresponding domain blocks are categorized depending on their smoothness parameter values (smoothness estimates), introduced, from the first, to characterize manifestation of high frequency components in the image. The searching of the best matched domain block is carried out between the neighbouring (or, within the same) smoothness classes. The computational complexity of the fractal image encoding process is reduced considerably. Theoretical and experimental investigations show that extremely high compression time savings are achieved for images of size $512 \times 512$.
\end{abstract}

\section{Introduction}

Those, who are closely bound up with digital images, acknowledge that the digital image processing problems, as well as image processing technologies, experience changes with the time flying by. There is no denying the fact that topics, such as detection of fractal nature of an image, image segmentation and texture analysis, synthesizing images, etc., came to the first place, [1-5]. The digital image analyzing tools have been expanding too - the new and promising mathematical means (discrete transforms, elements of fractal geometry, fundamental of genetics, etc.) were put at hand, [6-9].

In the field of digital images (computerized real-world image models) the fractal approach is of outmost importance, because it facilitates perception and understanding of the information content of an image. To say more, it provides us with a powerful means to catch sight of a fundamental real-world image property generally known as self-similarity. Due to this property, the research and development of algorithms (fractal techniques) to extract important fractal parameters from appropriate digital data has received significant attention in recent years. One of the most rapidly developing areas is the use of fractal geometry for performing image data compression.

Everybody, who is gone deep into the essence of the matter, comprehends that merely the extractability of self-similarity, found within images, made it possible to construct the fractal representation of an image. A. Jacquin was the first to propose a practical block based fractal image coding scheme (idea) in 1990, basis of most published fractal image coding schemes, $[2,5,10,11]$. 
However, the encoding complexity of the fractal image coding is extremely high and has become the major obstacle for its workable practical applications. In sum, the most computationally intensive part of the fractal encoding (compression) process is the searching step. If a brute force approach (full search) to the detection of optimal pairings "range block - domain block" is used, the fractal encoding complexity is always dominated by this searching process. The design of efficient domain search strategies (searching algorithms) has consequently been one of the most active areas of research in fractal coding, resulting in a wide variety of solutions. A survey of some really significant advances is represented in [10, 11]. Unfortunately, we have to emphasize that, despite numerous and many-sided attempts to accelerate fractal image encoding times, the "speed problem" so far remains to be unsolved. Naturally, any original proposal, any new idea, leading to the improvement of the overall performance of fractal image compression technologies, is worthy of great praise.

This paper introduces a new idea (strategy) to speeding up image compression, i.e., to overcoming the "speed problem" in the block-based fractal image coding procedures. The proposed idea rests on the direct application of invariant image smoothness parameter values - image smoothness estimates. The latter estimates are used in stating the necessary image similarity condition, which is employed later on to achieve image compression speed gains in the search for optimal pairings "range block-domain block". Theoretical and experimental analysis results show that the proposed strategy is comparatively simple in implementation and fast in encoding time, as compared with recently developed block based fractal compression schemes.

\section{Image Smoothness Estimates and Their Properties}

Consider a set of digital images $S^{2}(n)=\left\{[X(m)] \mid m=\left(m_{1}, m_{2}\right) \in I^{2}\right\}$, where: $I=\{0,1, \ldots, N-1\}, \quad N=2^{n}, \quad n \in \mathrm{N} ; \quad X(m) \in\left\{0,1, \ldots, 2^{p}-1\right\}$, for all $m \in I^{2}$; $p(p \geq 1)$ equals the number of bits per pixel in $[X(m)]$. The distance (mean squared error) $\delta$ between any two elements of the set $S^{2}(n)$ - images $\left[X_{1}(m)\right]$ and $\left[X_{2}(m)\right]$ is specified by.

$$
\delta=\delta\left(X_{1}, X_{2}\right)=\left(\frac{1}{N^{2}} \sum_{m \in I^{2}}\left(X_{2}(m)-X_{1}(m)\right)^{2}\right)^{1 / 2}
$$

Let us denote the two-dimensional discrete spectrum (Walsh-Hadamard (WHT), cosine (DCT), etc., [9]) of the image $[X(m)] \in S^{2}(n)$ by $\left[Y_{X}(k)\right], k=\left(k_{1}, k_{2}\right) \in I^{2}$. It is well known that the spectral coefficients $Y_{X}(k)$ decrease in absolute value, as their serial numbers $k$ (indices $k_{1}$ and $k_{2}$ ) increase, provided the basis vectors of the discrete transform in use are presented in a frequency order. The latter circumstance implies that there exists a hyperbolic surface

$$
z=z\left(x_{1}, x_{2}\right)=C /\left(x_{1} \cdot x_{2}\right)^{\alpha} \quad(C \geq 0, \alpha \geq 0),
$$


which approximates the ordered array of spectral coefficients $\left\{\left|Y_{X}(k)\right| \mid k=\left(k_{1}, k_{2}\right) \in I^{2}, k_{1}^{2}+k_{2}^{2} \neq 0\right\}$ in the mean squared error sense, i.e.,

$$
\delta=\delta\left(Y_{X}, z\right)=\left(\frac{1}{N^{2}-1} \sum_{\substack{k \in I^{2} \\\left(k_{1}^{2}+k_{2}^{2} \neq 0\right)}}\left(\left|Y_{X}(k)\right|-\frac{C}{\left(\bar{k}_{1} \cdot \bar{k}_{2}\right)^{\alpha}}\right)^{2}\right)^{1 / 2} \rightarrow \min ;
$$

here $\bar{k}_{i}=\max \left\{k_{i}, 1\right\}, \mathrm{i}=1,2$.

The quantity $\alpha$ (expression (2)), characterizing the shape of the hyperbolic surface, i.e., the rate of decay of spectral coefficients (high frequency components of the image), as their serial numbers increase, is assumed, in what follows, to be the smoothness parameter (level, class) of the image $[X(m)] \in S^{2}(n)$. This assumption is intuitively understandable - the more intense manifestation of high frequency components in the discrete spectrum of the image, the more noticeable changes of pixel intensity values (sharp edges) are detected in the image.

Below, we present a means for finding the very first approximation of the image smoothness parameter value $\alpha_{0}$. Let us designate the set of indices of nonzero spectral coefficients in the discrete spectrum $\left[Y_{X}(k)\right]$ of the image $[X(m)] \in S^{2}(n)$ as $H$, i.e.,

$$
H=\left\{k=\left(k_{1}, k_{2}\right) \in I^{2} \mid Y_{X}(k) \neq 0, k_{1}^{2}+k_{2}^{2} \neq 0\right\} .
$$

Then, application of the "linearization" procedure (logarithmization) both to the ordered array of nonzero spectral coefficients $\left\{\left|Y_{X}(k)\right| \mid k=\left(k_{1}, k_{2}\right) \in H\right\}$ and to the hyperbolic surface $z=C /\left(x_{1} \cdot x_{2}\right)^{\alpha}$ leads to the following result (objective function)

$$
\delta=\delta\left(\ln \left|Y_{X}\right|, \ln z\right)=\left(\frac{1}{|H|} \sum_{k=\left(k_{1}, k_{2}\right) \in H}\left(\ln \left|Y_{X}\left(k_{1}, k_{2}\right)\right|-\ln C+\alpha \ln \left(\bar{k}_{1} \cdot \bar{k}_{2}\right)^{2}\right)^{1 / 2} .\right.
$$

The minimum of the latter function is found using the least squares method (for the sake of simplicity, $\delta$ is squared), namely:

$$
\left\{\begin{array}{l}
\frac{\partial \delta^{2}}{\partial \ln C}=-\frac{2}{|H|} \sum_{k \in H}\left(\ln \left|Y_{X}\left(k_{1}, k_{2}\right)\right|-\ln C+\alpha \ln \left(\bar{k}_{1} \cdot \bar{k}_{2}\right)\right)=0, \\
\frac{\partial \delta^{2}}{\partial \alpha}=-\frac{2}{|H|} \sum_{k \in H}\left(\ln \left|Y_{X}\left(k_{1}, k_{2}\right)\right|-\ln C+\alpha \ln \left(\bar{k}_{1} \cdot \bar{k}_{2}\right)\right) \cdot \ln \left(\bar{k}_{1} \cdot \bar{k}_{2}\right)=0 .
\end{array}\right.
$$

Now, solving this system of linear algebraic equations (expression (6)) for $\alpha$, we easily derive 


$$
\alpha=\alpha_{0}=\frac{1}{A_{N}} \sum_{k \in H}\left(B_{N}-|H| \cdot P(k)\right) \cdot \log \left|Y_{X}(k)\right|,
$$

where: $\quad A_{N}=|H| \cdot C_{N}-B_{N}^{2} ; \quad B_{N}=\sum_{k \in H} P(k) ; \quad C_{N}=\sum_{k \in H} P^{2}(k) ; \quad P(k)=\log \left(\bar{k}_{1} \cdot \bar{k}_{2}\right)$, for all $k \in H$; by the way, $A_{N}=0$ if and only if the set $H$ is empty, i.e., the digital image $[X(m)]$ is absolutely smooth. It is worth emphasizing that the above "rough" image smoothness estimates (expression (7)), sometimes, serve the purpose.

To make the estimate more precise, various approaches can be applied, namely: successive coordinate optimization procedures, special iterative techniques, etc., [12]. Experimental results show that the real world image smoothness estimates, obtained using DCT, fall into the interval $(0 ; 3)$.

We have proved that the image smoothness parameter values (smoothness estimates) $\alpha$ for $[X(m)] \in S^{2}(n)$, found using DCT (or, WHT), possess the following exceptionally important properties, [12]:

1. Invariance of $\alpha$ with respect to some transformations (rotation, reflection, inversion, luminance change), acting upon the image $[X(m)]$.

2. Continuity of $\alpha: S^{2}(n) \rightarrow \mathrm{R}$, taken in the way that small (discrete) changes in $[X(m)]$ correspond to small (discrete) changes in $\alpha$.

Just the latter property makes it possible to introduce the necessary image similarity condition - two digital images $\left[X_{1}(m)\right] \in S^{2}(n)$ and $\left[X_{2}(m)\right] \in S^{2}(n)$ can not be similar if their smoothness parameter values (smoothness estimates $\alpha_{X_{1}}$ and $\alpha_{X_{2}}$, respectively) differ (in the sense of $\delta$ ) distinctly, i.e.,

$$
\left(\left|\alpha_{X_{1}}-\alpha_{X_{2}}\right|>\varepsilon_{0}\right) \Rightarrow\left(\delta\left(X_{1}, X_{2}\right)>\delta_{0}\right)
$$

here: $\delta_{0}$ is a small positive number (the threshold criterion value). For task-oriented applications, the relationship $\varepsilon_{0} \leftrightarrow \delta_{0}$, evidently, should be established experimentally.

\section{Speeding Up Fractal Image Compression}

Since A. Jacquin described the first practical block based fractal image coding scheme, [2], fractal image coding technique has attracted a lot of attention as a new digital image processing technology. However, the encoding complexity of the fractal image coding is very high and has become the major obstacle for its workable applications. It is well known that the most computationally intensive part of the fractal encoding (compression) process is the searching step. This is required for each range block to find the best matched domain block within the searching region (pool). If the brute force strategy (full search; Jacquin's approach) is used, computation, needed to perform fractal encoding, is enormous ("speed problem"). Over the past 
years, many fast searching algorithms have been developed to surmount the "speed problem", [5, 10, 11].

We here present a new attempt (idea, strategy) to improve compression times in block based fractal image coding procedures (Figure 1). Let $[X(m)] \in S^{2}(n)$ be an image to be processed. Fractal image compression speed gains are achieved, mainly, owing to the following factors:

1. Firstly, for the determination of smoothness level, each range block $\left[U_{i}(m)\right] \in S_{1}^{2}\left(n_{1}\right) \subset S^{2}\left(n_{1}\right), \quad$ as well as each domain block $\left[V_{j}(m)\right] \in S_{1}^{2}\left(n_{2}\right) \subset S^{2}\left(n_{2}\right)$, is looked over only once (here: $i=1,2, \ldots, 4^{n-n_{1}}$; $j=1,2, \ldots,\left(2^{n}-2^{n_{2}}+1\right)^{2}$; usually, $n_{1} \in\{2,3\}$ and $\left.n_{2}=n_{1}+1\right)$. As a result, two sequences of image smoothness estimates, $\left\{\alpha_{U_{i}}\right\}$ and $\left\{\alpha_{\hat{V}_{j}}\right\}$, are formed $\left(\left[\hat{V}_{j}(m)\right] \in S^{2}\left(n_{1}\right)\right.$ is a shrunken copy of the domain block $\left.\left[V_{j}(m)\right]\right)$. Thus, domain and range blocks are categorized into a finite number of classes, according to their invariant representations - smoothness parameter values (smoothness estimates).

Now, direct distance comparisons between the smoothness estimates make it possible to determine the best pairings "range block - domain block". The search region (domain pool) for a particular range block $[U(m)]$ is limited by the upper bound $\varepsilon_{0}$ for the difference $\left|\alpha_{U}-\alpha_{\hat{V}}\right|$ (the necessary image similarity condition; Section 2).

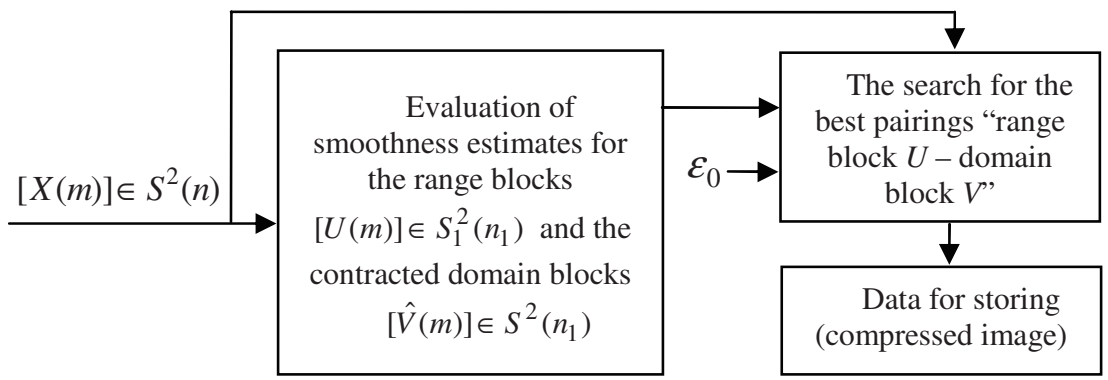

Fig. 1. Fractal image encoding scheme - implementation of the necessary image similarity condition

2. Secondly, a thorough analysis of algebraic expressions and actions used in evaluating the DCT spectrum of the range block (or, contracted domain block; $n_{1}=3$ ), in finding image (block) smoothness estimates, as well as in establishing the fact of block similarity, shows that the total time expenditures $(\tau)$, associated with these steps, are equal to

$$
\tau=\left(\frac{9709}{64} N^{2}+10029(N-15)^{2}+\frac{553}{8} N^{2} M\right) \cdot \tau_{a},
$$


provided the time expenditures, required to perform a single addition and multiplication operations, are equal, i.e., $\tau_{a}=\tau_{m}$; here $M$ indicates the averaged number of domain blocks $[V(m)] \in S_{1}^{2}(4) \subset S^{2}(4)$ contained in the search region (pool) related to the range block $[U(m)] \in S_{1}^{2}(3) \subset S^{2}(3) ; N \times N$ is the size of the image under processing.

Similarly, the total time expenditure $\left(\tau^{\circ}\right)$, associated with the brute force strategy (Jacquin's approach), equals

$$
\tau^{\circ}=\left(\frac{65}{64} N^{2}+65(N-15)^{2}+\frac{553}{8} N^{2}(N-15)^{2}\right) \cdot \tau_{a} .
$$

Now, fractal image compression (encoding) time savings can be expressed in terms of $k=\tau^{\circ} / \tau$ (Figure 2). In particular, for $N=512$, the ratio $k$ exceeds 200, provided $M \leq 10^{3}$; theoretically, for $M \leq 10^{4}$, "success" (compression time savings) is ensured unconditionally.

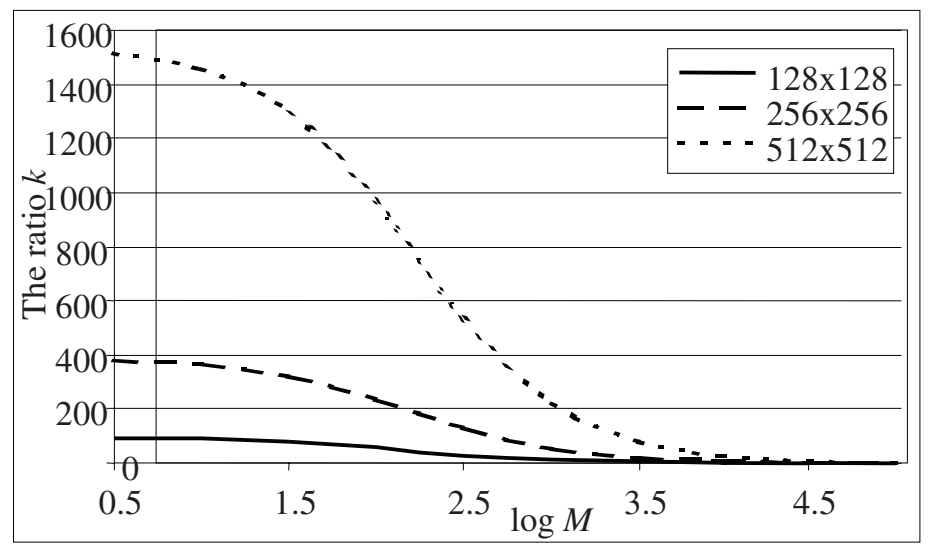

Fig. 2. Comparative analysis of fractal image encoding complexity $\left(k=\tau^{\circ} / \tau\right)$

But, what are the really acceptable values of $M$ ? Experimental results show that those values, as well as the quality of restored images, depend on both the upper bound $\varepsilon_{0}$ (expression (8); Section 2) and the smoothness level of range blocks.

\section{Experimental Results}

To corroborate the obtained theoretical results (Sections 2 and 3), some test images, characterized by different smoothness parameter values, were analysed (Figure 3). The range blocks were chosen to be of size $4 \times 4$, and the domain blocks - of size $8 \times 8$. Smoothness estimates were found using DCT. 


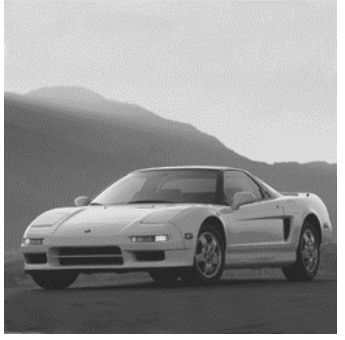

(a)

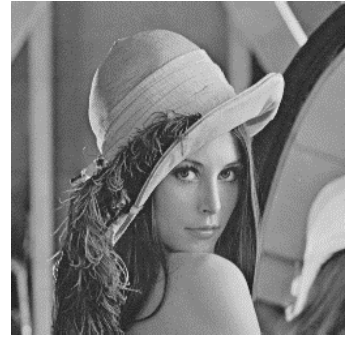

(b)

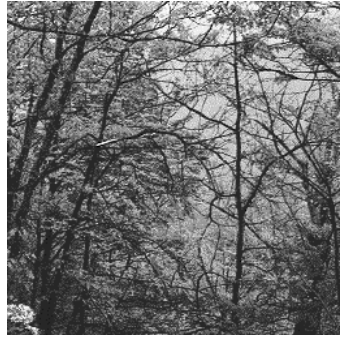

(c)

Fig. 3. Test images: (a) image "Acura" 256x256, $\alpha=1.44$; (b) image "Lena" 256x256, $\alpha=0.69$; (c) image "Forest" 256x256, $\alpha=0.37$

Table 1 contains experimental results, associated with the averaged number $M$ of domain blocks in a pool (expression (9); Section 3), for the test images "Acura", "Lena" and "Forest" (Figure 3). It can be seen that the averaged pool size variations highly depend on the smoothness level of the image under processing.

Comparative analysis of two strategies - the full search for optimal pairings (brute force approach), characterized by the mean squared error $\delta_{\text {opt }}$, and the proposed search strategy, based on the use of image smoothness estimates and characterized by the mean squared error $\delta_{\varepsilon_{0}}$, is presented, also, in Table 1. Analysis results show, the higher smoothness of the image under processing, the better pairing results (in the sense of $\delta_{\varepsilon_{0}}$ ) are obtained.

On the other hand, if the value $\varepsilon_{0}$ (the upper bound for the difference $\left|\alpha_{U}-\alpha_{V}\right|$ ) is chosen, say, to be not less than 0.1 , then all the selected (best) pairings "range block - domain block" will appear to be sufficiently close to the optimal ones (Table 1).

The overall performance (compression speed gains) of the proposed fractal image encoding strategy is shown in Table 2 (Computer simulation was performed on a PC with CPU AMD1800+(@2800+), RAM 512MB, OS Windows XP).

Table 1. Dependence of the averaged pool size $M$ and the averaged deviation $\Delta \delta=\delta_{\varepsilon_{0}}-\delta_{\text {opt }}$ on $\varepsilon_{0}$

\begin{tabular}{ccccccc}
\hline $\begin{array}{c}\text { The } \\
\text { upper } \\
\text { bound, }\end{array}$ & \multicolumn{2}{c}{$\begin{array}{c}\text { The averaged number of domain } \\
\text { blocks in a pool, } M\end{array}$} & \multicolumn{2}{c}{ The averaged deviations, $\Delta \delta$} \\
$\varepsilon_{0}$ & "Acura" & $\begin{array}{c}\text { Image } \\
\text { "Lena" }\end{array}$ & $\begin{array}{c}\text { Image } \\
\text { "Forest" }\end{array}$ & $\begin{array}{c}\text { Image } \\
\text { "Acura" }\end{array}$ & $\begin{array}{c}\text { Image } \\
\text { "Lena" }\end{array}$ & Image \\
\hline 0.001 & 63 & 51 & 94 & 1.76021 & 2.54327 & 4.76107 \\
0.005 & 340 & 294 & 817 & 0.96680 & 1.50931 & 3.14990 \\
0.010 & 682 & 589 & 1721 & 0.71430 & 1.25823 & 2.55641 \\
0.020 & 1385 & 1204 & 3527 & 0.57229 & 1.01700 & 2.03292 \\
0.040 & 2787 & 2438 & 7101 & 0.42149 & 0.81976 & 1.57958 \\
0.100 & 6966 & 6123 & 17340 & 0.25324 & 0.49752 & 0.89875 \\
0.150 & 10343 & 9129 & 25088 & 0.18624 & 0.37377 & 0.60599 \\
\hline
\end{tabular}


It can be seen, that quite tolerable (in the sense of $\delta=\delta(X, \tilde{X})$; here $[\tilde{X}(m)]$ is the restored image) processing results are obtained even for $\varepsilon_{0} \leq 0.005$ (images "Acura" and "Lena"; Table 2). In such cases, compression time savings are sufficiently good ( $k=\tau^{\circ} / \tau>18$, for "Acura", and $k>60$, for "Lena"; Table 2).

Table 2. Fractal image compression speed gains and the quality of restored images, for different values of $\varepsilon_{0}$

\begin{tabular}{|c|c|c|c|c|c|c|}
\hline \multirow{2}{*}{$\begin{array}{c}\text { The } \\
\text { upper } \\
\text { bound, } \\
\varepsilon_{0}\end{array}$} & \multicolumn{3}{|c|}{$\begin{array}{l}\text { The quality of restored images, } \\
\qquad \delta=\delta(X, \tilde{X})\end{array}$} & \multicolumn{3}{|c|}{ The total time expenditure, $\tau$} \\
\hline & $\begin{array}{l}\text { Image } \\
\text { "Acura", }\end{array}$ & $\begin{array}{l}\text { Image } \\
\text { "Lena" }\end{array}$ & $\begin{array}{l}\text { Image } \\
\text { "Forest", }\end{array}$ & $\begin{array}{l}\text { Image } \\
\text { "Acura" }\end{array}$ & $\begin{array}{l}\text { Image } \\
\text { "Lena", }\end{array}$ & $\begin{array}{l}\text { Image } \\
\text { "Forest" }\end{array}$ \\
\hline $\begin{array}{c}\text { Full } \\
\text { search }\end{array}$ & 2.41 & 3.80 & 17.88 & 547.06 & 550.38 & 546.98 \\
\hline 0.200 & 2.87 & 4.27 & 19.42 & 330.95 & 213.65 & 164.13 \\
\hline 0.100 & 3.03 & 4.44 & 20.22 & 242.50 & 121.59 & 84.19 \\
\hline 0.050 & 3.27 & 4.64 & 21.12 & 164.81 & 66.34 & 43.05 \\
\hline 0.013 & 3.68 & 5.09 & 23.00 & 67.53 & 19.48 & 12.39 \\
\hline 0.005 & 4.02 & 5.79 & 24.38 & 30.39 & 8.61 & 5.65 \\
\hline 0.001 & 5.20 & 7.23 & 27.57 & 8.69 & 3.11 & 2.31 \\
\hline
\end{tabular}

More impressive fractal image compression speed gains are obtained for images of size $512 \times 512$ (Figure 4; image "Maroon"). For instance, the upper bound value $\varepsilon_{0}=0.005$ ensures very high compression time savings $(k>145)$ and, above all, the quality of the restored image $[\tilde{X}(m)]$ remains to be sufficiently good (Figure $4, \mathrm{~b}, \mathrm{e})$.

\section{Conclusion}

Theoretical and experimental analysis results confirm usefulness of the proposed image compression time accelerating approach, based on the use of image smoothness estimates. Compression time savings are achieved, mainly, owing to the following two factors: firstly, for the determination of the level of smoothness, each domain block, as well as each range block, is looked over only once; secondly, candidate domain blocks (forming a pool) and corresponding range block, roughly speaking, fall into the same class of smoothness. So, to ensure fast search for best pairings, it is quite enough to analyze only a small number of pairs "range block-domain block". The quality of restored images satisfies the needs too.

In spite of the fact that fractal image coding techniques (the basic Jacquin's idea and plenty of its modifications), based on the use of partitioned iterated function systems and distinguishing themselves by a clear disproportion of time expenditures, needed to perform image encoding and image decoding steps, cannot (till now) compete with the most widely used in practice the still image compression standard 
JPEG (basically, at lower compression ratios), any attempt to promote (modify, improve) the very promising fractal image processing technologies, is worthy of great praise.

Future research we are to concentrate on two-dimensional binary (white-and-black) images, on the determination of their smoothness parameter values and on the development of appropriate fractal image coding schemes.

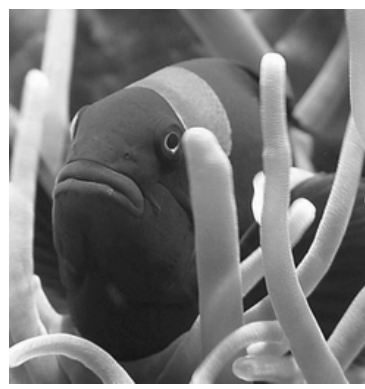

(a)

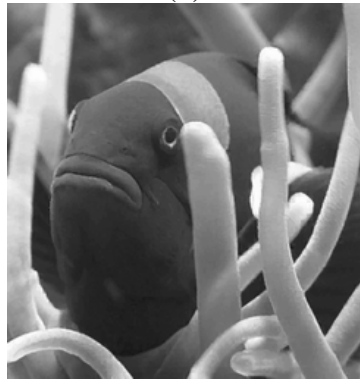

(d)

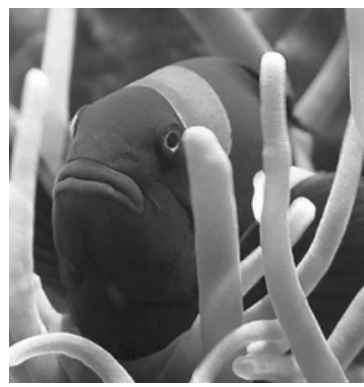

(b)

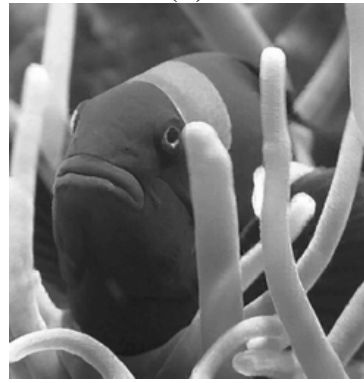

(e)

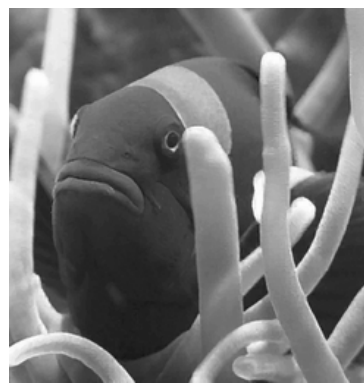

(c)

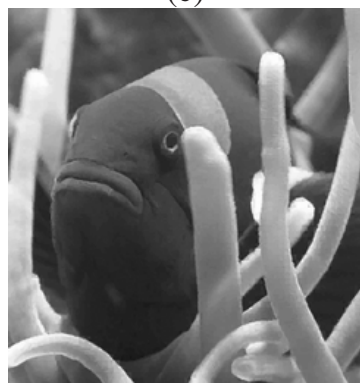

(f)

Fig. 4. Fractal image compression speed gains (range blocks $8 \times 8$, domain blocks $16 \times 16$ ): (a) image „Maroon“ 512x512; (b) full search ( $\tau=6132.14 \mathrm{sec}, \delta=4.31$ ); (c) $\varepsilon_{0}=0.025$, $\tau=172.72 \mathrm{sec}, \delta=4.90$ ); (d) $\varepsilon_{0}=0.013, \tau=94.59 \mathrm{sec}, \delta=5.09$; (e) $\varepsilon_{0}=0.005, \tau=42.16 \mathrm{sec}$, $\delta=5.39 ;$ (f) $\varepsilon_{0}=0.001, \tau=17.95 \mathrm{sec}, \delta=6.24$

\section{References}

1. Peitgen, H.-O., Jurgens, H., Saupe, D.: Chaos and Fractals. Springer-Verlag (1992)

2. Jacquin, A.: Image Coding Based on a Fractal Theory of Iterated Contractive Image Transformations. IEEE Transactions on Image Processing, Vol. 1, no. 1 (1992) 18-30

3. Turner, M. J., Blackledge, J. M., Andrews, P. R.: Fractal Geometry in Digital Imaging. Academic Press, Cambridge (1998)

4. Valantinas, J., Zumbakis, T.: On the Use of Shift Dynamics in Synthesizing Fractal Images. Intern. Journ. INFORMATICA, Vol. 15, no. 3. Institute of Mathematics and Informatics, Vilnius (2004) 411-424

5. Fisher, Y.: Fractal Image Compression - Theory and Application. Springer-Verlag New York (1994) 
6. Wallace, G.K. : The JPEG Still Picture Compression Standard. Communications of the ACM, Vol. 34, no. 4 (1991) 30-44

7. Culik II, K., Valenta, V.: Finite Automata Based Compression of Bi-Level and Simple Color Images. Department of Computer Science, University of South Carolina, Columbia , S.C. 29208, U.S.A (1998) 1-14

8. Valantinas, J., Valantinas, R.: Problem-Oriented Change of Image Dimensionality. Proceedings of the Third International Symposium on Image and Signal Processing and Analysis, Rome (Italy), Universita degli Studi ROMA TRE (2003) 228-232

9. Ahmed, N., Rao, K.R., Orthogonal Transforms for Digital Signal Processing. SpringerVerlag, Berlin Heidelberg New York (1975)

10. Saupe, D., Hamzaoui, R.: Complexity reduction methods for fractal image compression. Proceedings of the IMA Conference on Image Processing: Mathematical Methods and Applications, Oxford, England (1994) 211-229

11. Wohlberg, B., de Jager, G.: A review of the Fractal Image Coding Literature. IEEE Transactions on Image Processing, Vol. 8, no. 12 (1999) 1716-1729

12. Valantinas, J., Žumbakis, T.: Definition, evaluation and task-oriented application of image smoothness estimates. Information Technology and Control, no. 1(14), Technologija, Kaunas (2004) $15-24$ 